Journal of Fundamental and Applied Sciences

ISSN 1112-9867

Available online at

http://www.jfas.info

\title{
STUDY ON THE ENERGETIC PARAMETERS IN A PHOTOTHERMIC SENSOR WITH BLACK POLYMERIC FILM
}

\author{
A. Benmoussat ${ }^{*}$, N. Baba Ahmed, S. Ainad Tabet and B. Belahcene \\ Engineering Faculty, Abou Bekr Belkaid University - Tlemcen BP 230 -Chetouan \\ 13000 - Tlemcen Algérie
}

Received: 01 February 2010 / Accepted: 02 June 2010 / Published online: 30 June 2010

\begin{abstract}
Renewable energies such as thermal solar energy are accessible energy hydrous for desalination water, recycling and hot water production. Heat is produced by greenhouse effect in the sensor. It is no polluting form of energy according to the sustainable development.

In this paper the design of sensor photothermic prototype with polymeric film absorbing, the materials radiation properties, the analysis of different energy losses in the system and the energy balance will be presented. The evolution of incidental solar illumination on the horizontal plan of sensor and the temperature distribution are studied.
\end{abstract}

Results showed that the temperature obtained by thermal conversion depends on the absorbing characteristics of the polymeric film, radiation intensity and time exposure.

Keys words: film, solar energy, greenhouse effect, design, radiation, illumination.

\section{INTRODUCTION}

La demande croissante en eau douce pour les populations, l'agriculture et l'industrie devient un problème qui s'accentue quand la sécheresse peut sévir pendant des années. L'eau n'est pas répartie selon les besoins de chaque région, certaines régions sont affectées de pénuries chroniques comme le désert alors que d'autres affichent des surplus [1].

Author Correspondence, e-mail: abbenmoussa@gmail.com

ICID: 1037248 
La distillation par effet de serre est l'une des applications énergétiques du solaire thermique à basse température qui consiste à la conversion de l'énergie solaire (rayonnement) en énergie thermique pour produire de l'eau à partir d'une eau saline, où elle s'adapte en mode individuel ou collectif à des régions éloignées ou isolées qui nécessite des consommations réduites [2]. Le choix des matériaux absorbants du capteur thermique en augmentant leurs caractéristiques d'absorption conditionne le rendement énergétique avec le choix des matériaux transparents [3].

Nous sommes intéressés dans ce travail à l'étude les paramètres énergétiques de la distillation solaire et des transferts thermiques résultant par suite de la conversion du rayonnement solaire en chaleur par effet de serre dans un prototype de distillateur à plan incliné à surface d'évaporation horizontale réalisé dans notre laboratoire à base d'absorbeur en film noir polymère et de vitrage transparent en verre non traité.

Le dimensionnement du capteur, l'évolution de l'éclairement solaire incident, les variations du taux de salinité, la hauteur de l'eau saline admise dans le capteur, le système d'isolation, l'évolution de la température en différents points du distillateur, la température ambiante et la quantité de l'eau distillée sont étudiés et nous terminerons par une modélisation de la distribution de la température dans le système étudié.

\section{MATERIELS ET METHODES}

\section{Description et principe de fonctionnement du capteur}

La figure (1) présente un prototype de distillateur à plan incliné, il est constitué d'un bac ouvert sur le coté exposé aux rayonnements solaires, le fond et les parois latérales sont isolés par une couche isolante. Le bac est fermé au dessus par un vitrage incliné d'un certain angle par rapport à l'horizontal, jouant le rôle de surface de condensation. Les radiations solaires qui traversent le vitrage sont absorbées par le polymère noir qui réémet l'énergie sous forme de radiations de grande longueur d'onde. Comme ces radiations ne peuvent pas traverser la vitre, la température de la plaque d'absorption s'élève et chauffe la masse d'eau contenue dans le bac et fait évaporer sa couche superficielle. Au moyen de la convection naturelle la vapeur de l'eau s'élève est arrive au niveau de la surface intérieur du vitrage où la température de ce dernier est relativement inférieure par rapport à celle de la vapeur, et donc se condense. Les gouttelettes d'eau condensées glissent sur la surface inclinée du vitrage intérieure et se rassemblent dans un canal de récupération (rigole). 
Pour obtenir un meilleur rendement, l'ensemble est placé dans une boîte vitrée isolante afin d'obtenir un effet de serre. Avec un ensoleillement important, et si les besoins en énergie sont modérés, l'absorbeur, est chauffé par le rayonnement solaire et transmet la chaleur à l'eau qui s'évapore et se condense sur la partie inférieure de la vitre transparente. Bien que le distillateur soit d'une construction très simple, et peu coûteuse, son rendement en matière d'eau distillée reste encore très faible c'est pourquoi la conception d'autres modèles de distillateurs ont été envisagés en vue d'augmenter le rendement.

Compte tenu des moyens dont nous disposons au laboratoire, nous avons réalisés ce prototype à partir de matériaux disponibles en commerce

Tableau 1. Caractéristiques des matériaux isolants et des matériaux transparents

\begin{tabular}{|c|c|c|c|}
\hline $\begin{array}{c}\text { Isolant } \\
\text { (Bois sec ordinaire) }\end{array}$ & Dimensions & $\begin{array}{c}\text { Vitrage } \\
\text { (vitre ordinaire) }\end{array}$ & \\
\hline Epaisseur du bois & $0.008 \mathrm{~m}$ & Nombre & 1 \\
\hline Surface intérieure & $0.69 \mathrm{~m}^{2}$ & Epaisseur de la vitre & $0.59 \mathrm{~mm}$ \\
\hline (base du boîtier) & & Inclinaison de la vitre & $18^{\circ}$ \\
\hline Hauteur arrière du boîtier & $0.345 \mathrm{~m}$ & Dimension de la surface & $0.323 \times 0.74 \mathrm{~m}^{2}$ \\
\hline Hauteur avant du boîtier & $0.12 \mathrm{~m}$ & & \\
\hline Surface d'évaporation & $0.31 \times 0.69 \mathrm{~m}^{2}$ & & \\
\hline
\end{tabular}

Nous avons utilisé deux types d'isolations successivement pour renforcer l'action du bois qui présente un coefficient de conductibilité un peu élevé $(\lambda=0.13$ à $0.40 \mathrm{~W} / \mathrm{m} . \mathrm{K})$; et par conséquent une perte de chaleur relativement élevée; une peinture blanche appliquée sur toute la surface extérieure du bois pour les premiers essais et un film de feuille d'aluminium fin ayant un pouvoir réflecteur élevé $((\rho=0.95)$ afin de limiter les pertes par convection thermique de la surface chaude qui sera superposé par un film de plastique transparent pour les seconds essais. Ce dernier servant de support résistant pour l'aluminium très fragile et contribuant en même tant par ses propriétés de mauvais conducteur thermique.

Les matériaux absorbeurs utilisés sont la peinture noire du commerce que nous avons appliqué sur la base interne du capteur et un film polymère noir disponible sur le commerce. Ce dernier jouant le rôle d'absorbeur et de membrane étanche. Il est déposé au fond du capteur de telle sorte qu'il épouse sa forme. 


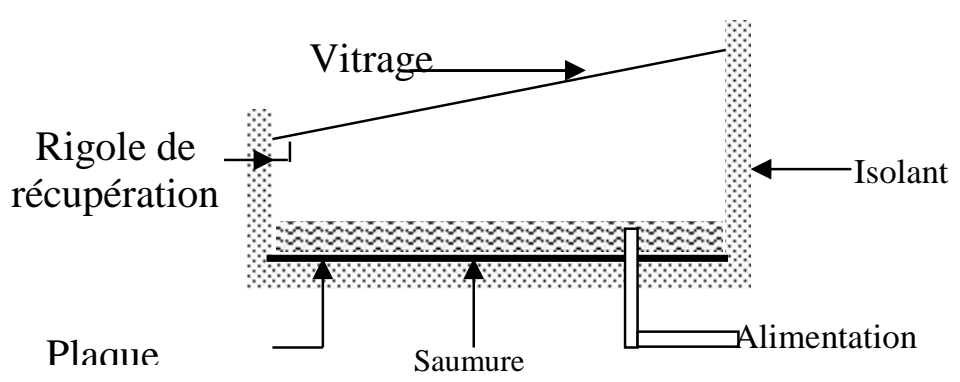

Fig.1a. Schéma du distillateur solaire plan incliné

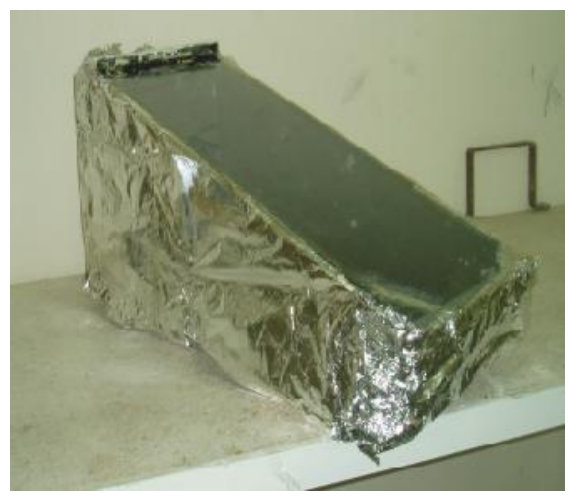

Fig.1b. Distillateur solaire réalisé

L'énergie thermique disponible à partir d'un tel capteur est équivalente à l'énergie incidente diminuée des pertes dues à la réflexion, la réémission du rayonnement, la convection et la conduction. Toute utilisation du rayonnement solaire doit prendre en compte les diverses caractéristiques du rayonnement incident telle que les variations instantanées, horaire et journalière, et annuelle de l'éclairement qui présente une dépendance vis à vis des caractéristiques du climat local et régional [3]. Pour le site de Tlemcen, les coordonnées géographiques sont : Altitude : $750 \mathrm{~m}$, Latitude $+35^{\circ} 28^{\prime} \mathrm{N}$ et Longitude $-1^{\circ} 17^{\prime}$

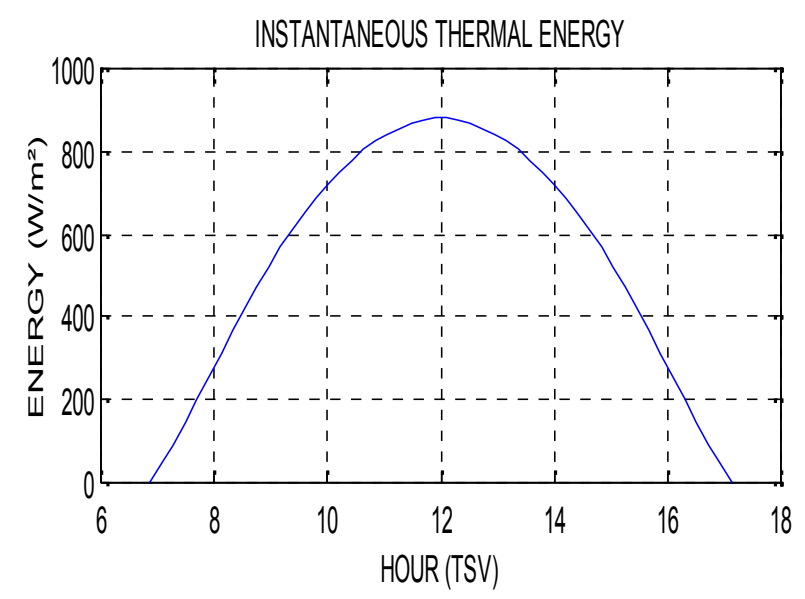

Fig.2. Radiations solaires incidentes sur le plan horizontal

\section{Tests}

Le prototype réalisé a été installé sur un plan horizontal et orienté en plein sud pour avoir un maximum d'ensoleillement (figure1b). Les tests ont été effectués sur le terrain contigu au laboratoire pendant la période allant du 15 au 26 mai 2009. L'eau saumâtre 
à été préparée au laboratoire avec des concentrations en sel $\mathrm{NaCl}$ allant de 5 à $10 \mathrm{~g} / \mathrm{l}$. Le distillateur a été rempli à des hauteurs variant de 1 à $5 \mathrm{~cm}$. La campagne de tests a été axée sur les mesures de la température par thermocouple de type $\mathrm{K}$ et par thermomètre digital pour les mesures de la température de la vitre et de l'isolant et du débit de l'eau distillée en fonction du temps.

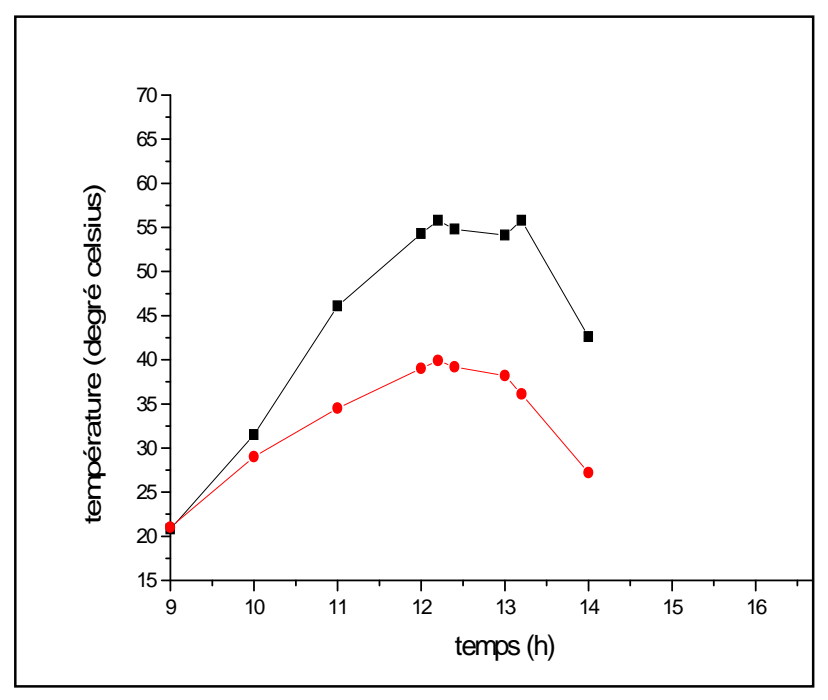

$3 \mathrm{a}$

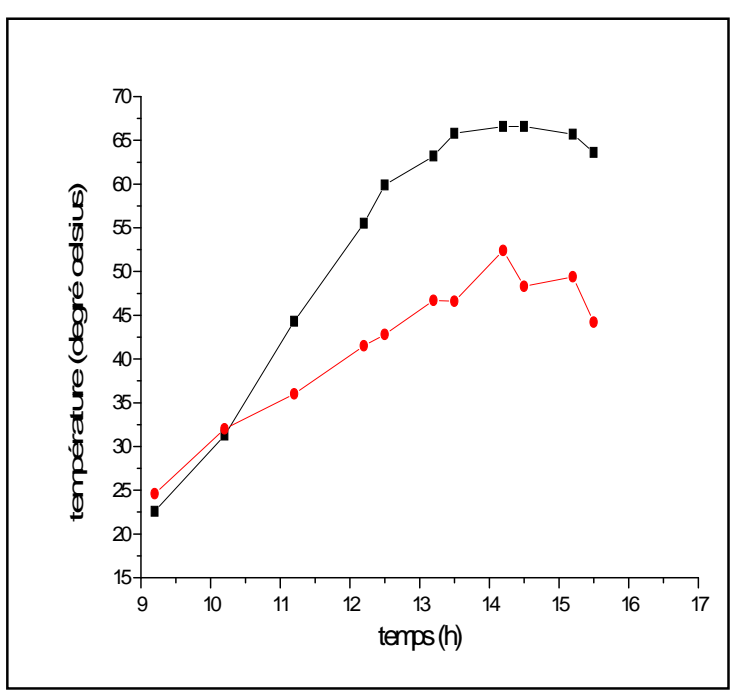

$3 b$

Fig.3. Evolution des températures de l'absorbeur en film polymère et de la vitre non traitée en fonction du temps montrant l'effet de l'isolation thermique externe ( $3 \mathrm{a}$ sans réflexion, $3 \mathrm{~b}$ avec réflexion), $(\mathrm{h}=1$ à $3 \mathrm{~cm}$ et $\mathrm{C}=5$ à $10 \mathrm{~g} / \mathrm{l})$

Tableau 2. Journée du 30 mai 2009

\begin{tabular}{|c|c|c|c|c|c|c|c|}
\hline \multicolumn{8}{|c|}{$\mathrm{h}=3 \mathrm{~cm} ; \mathrm{C}=10 \mathrm{~g} / \mathrm{l}$} \\
\hline temps & $\mathrm{Te}\left({ }^{\circ} \mathrm{C}\right)$ & Ta $\left({ }^{\circ} \mathbf{C}\right)$ & $\mathbf{T v}\left({ }^{\circ} \mathbf{C}\right)$ & $\mathbf{T}_{\mathbf{i}}\left({ }^{\circ} \mathbf{C}\right)$ & $\mathbf{Q}(\mathrm{ml})$ & état ciel & Vent \\
\hline $9 \mathrm{~h} 20$ & 25,3 & 22,6 & 24,6 & $\mathrm{X}$ & 0 & Clair & Calme \\
\hline $10 \mathrm{~h} 20$ & 26,3 & 31,3 & 32 & 29,1 & 0 & Clair & Calme \\
\hline $11 \mathrm{~h} 20$ & 29,5 & 44,3 & 36 & 37,5 & 3 & Clair & Calme \\
\hline $12 \mathrm{~h} 20$ & 32,1 & 55,5 & 41,5 & 37,8 & 20 & Clair & Calme \\
\hline $12 \mathrm{~h} 50$ & 30,3 & 59,9 & 42,8 & 37,5 & 27 & Clair & Calme \\
\hline $13 \mathrm{~h} 20$ & 31 & 63,2 & 46,7 & 39,1 & 35 & Clair & Calme \\
\hline $13 \mathrm{~h} 50$ & 30,7 & 65,8 & 46,6 & 39,9 & 38 & Clair & Calme \\
\hline $14 \mathrm{~h} 20$ & 32,4 & 66,6 & 52,4 & 48,6 & 39 & Clair & Calme \\
\hline $14 \mathrm{~h} 50$ & 31,6 & 66,6 & 48,3 & 43 & 44 & Clair & Calme \\
\hline $15 \mathrm{~h} 20$ & 31,4 & 65,7 & 49,4 & 43,2 & 45 & Clair & Calme \\
\hline $15 \mathrm{~h} 50$ & 31,5 & 63,6 & 44,2 & 39,7 & 42 & Clair & Calme \\
\hline
\end{tabular}


- $\mathrm{T}_{\mathrm{e}}$ : température ambiante du milieu extérieur $\left({ }^{\circ} \mathrm{C}\right)$,

- $\mathrm{T}_{\mathrm{a}}$ : température de 1 'absorbeur $\left({ }^{\circ} \mathrm{C}\right)$,

- $\mathrm{T}_{\mathrm{v}}$ : température de la face externe de la vitre $\left({ }^{\circ} \mathrm{C}\right)$,

- C : concentration de l'eau saumâtre $(\mathrm{g} / \mathrm{l})$.

\section{Modélisation de la distribution de la température}

Les phénomènes de transfert de chaleur pure et de transfert simultané de chaleur et de masse à l'intérieur et à l'extérieur du capteur, chauffé par un flux solaire global constant et distillant une charge d'eau pure maintenu constante montre que la température n'est pas uniformément répartie selon les dimensions du capteur et dépend de l'isolation externe, comme le montre la figure 4. Pour le système sans réflexion de l'aluminium, la température oscille autour de $300 \mathrm{~K}$ en moyenne au niveau de la face avant de la vitre, tandis que pour un système avec réflexion la température est de l'ordre de $330 \mathrm{~K}$ en moyenne.

Pour notre dispositif, nous avons obtenu la répartition axiale de la distribution de la température avec une modélisation par les éléments finis en utilisant le logiciel Quick Field [4]

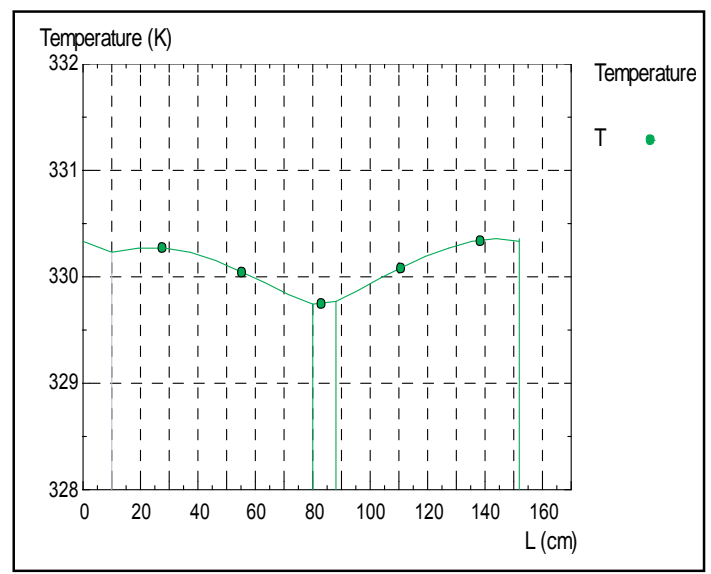

$4 \mathrm{a}$

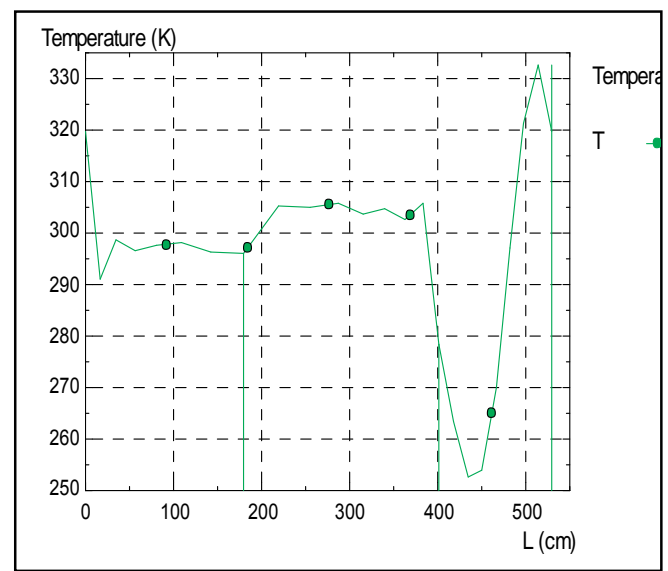

$4 \mathrm{~b}$

Fig.4. Distribution de la température $\left({ }^{\circ} \mathrm{K}\right)$ en fonction de la largeur $\mathrm{L}(\mathrm{cm}) \mathrm{du}$ distillateur $(4 \mathrm{a}-$ sans isolation en film aluminium, $4 \mathrm{~b}$ - avec isolation en film aluminium) 


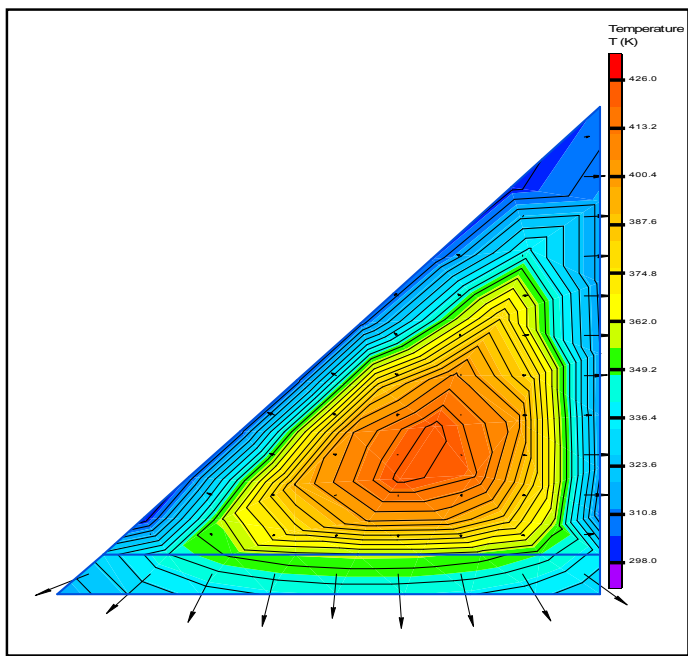

$5 \mathrm{a}$

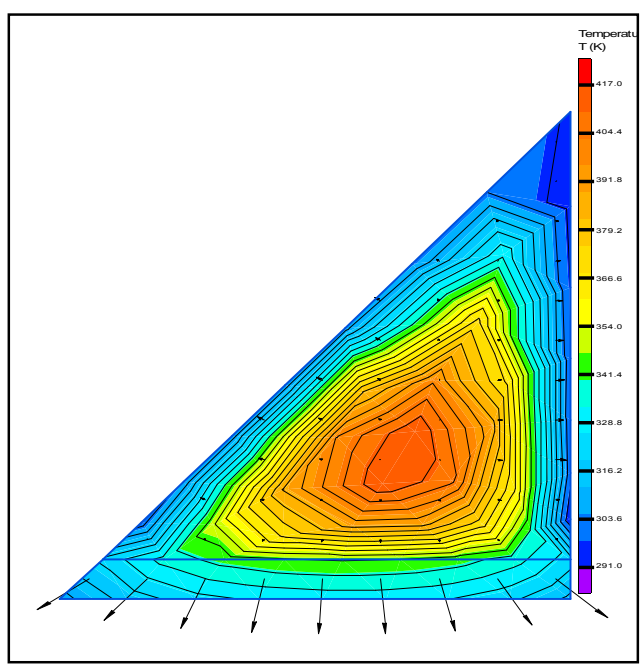

$5 b$

Fig.5. Répartition axiale de la distribution de la température

(5a sans réflexion par film d'aluminium - 5b avec réflexion par film d'aluminium)

\section{RESULTATS ET DISCUSSIONS}

\section{Tests de distillation}

L'énergie solaire reçue en un point du globe dépend de la latitude du lieu, ainsi vers l'équateur l'angle d'incidence est proche de $90^{\circ}$ donc la surface éclairée est plus petite et l'énergie est plus concentrée, et dépend aussi de la nébulosité (nuages), qui est importante à l'équateur et plus faible en milieu intertropical. Le potentiel solaire en Algérie [4] estimé en énergie moyenne reçue $\left[\mathrm{KWh} / \mathrm{m}^{2} / \mathrm{an}\right]$ est de 1700 en régions côtières et de 2650 au Sahara est l'un des meilleurs gisements au monde non encore exploité.

Les tests de distillation solaire ont été effectués suivant des conditions météorologiques favorables en exposition solaire en cette période de l'année (ciel clair avec vent à faible vitesse), les températures maximales qu'on peut atteindre dans le modèle réalisé compte tenu du système d'isolation et des déperditions sont de $1^{\prime}$ ordre $45.7^{\circ} \mathrm{C}$ à $13 \mathrm{~h} 20$ et $52.4^{\circ} \mathrm{C}$ à $14 \mathrm{~h} 20$ sur le vitrage et de l'ordre de $56.3^{\circ} \mathrm{C}$ à $15 \mathrm{~h} 25$ et $66.6^{\circ} \mathrm{C}$ à $14 \mathrm{~h} 50$ au niveau de l'absorbeur respectivement pour les deux journées (19 mai et 30 mai 2009). Ces températures ont été obtenues au temps TSV.

Cette différence de température entre l'absorbeur et le vitrage conduit au fonctionnement du capteur elle est d'autant plus grande que la quantité d'eau distillée produite est plus grande. Nous avons constaté qu'a des périodes où le vent soufflant 
même à faible vitesse contribue au refroidissement de la vitre et par conséquent à une diminution de la température, ce qui accentue le processus de condensation de vapeur. L'absorbeur en film polymère est un corps noirs absorbant théoriquement toute l'énergie électromagnétique qu'il recevait dont le spectre ne dépend que de sa température. $\mathrm{Vu}$ la mince couche du polymère en contact avec la couche de peinture noire, nous n'avons pas atteint des températures élevées où il a été suggéré d'interposer une couche d'une tôle en cuivre présentant de meilleurs caractéristiques d'absorption. Les données sur les quantités d'eau produite présentent une certaine dispersion. Pour chaque nuage de points nous avons déterminé une courbe de tendance polynomiale d'ordre 4. Nous avons obtenus un maximum de débit pour une exposition solaire après le temps TSV, un système d'isolation renforcé de l'extérieure par le film d'aluminium qui joue un rôle important dans la réflexion des rayons solaires; et une diminution de la hauteur de la nappe à distiller avec une hauteur optimale permet un échange thermique. Ce qui permet au système de fonctionner encore en période nocturne avant de s'annuler. La salinité de l'eau saumâtre pour des teneurs titrant moins que $10 \mathrm{~g} / \mathrm{l}$ ont une influence négligeable sur le fonctionnement du système.

\section{Distribution de la température}

Les résultats des essais ont montrés que la température n'est pas uniformément répartie selon les dimensions du capteur et dépend de l'isolation externe. La figure 5a représente une répartition axiale de la distribution de la température tout au long du distillateur pour un essai sans aluminium isolant qui montre une concentration de la température dans la zone tampon. Aux parois du distillateur la température est de l'ordre de $300 \mathrm{~K}$, elle augmente au fur et à mesure quelle se concentre vers le milieu tampon, elle est de l'ordre de $417 \mathrm{~K}$, et pour la figure $5 \mathrm{~b}$ avec un essai avec aluminium isolant la répartition axiale de la distribution de la température tout au long de distillateur montre une concentration de la température dans la zone tampon. La température du milieu tampon est de l'ordre de $426 \mathrm{~K}$.

Ces résultats sont en accord avec les résultats expérimentaux, malgré un décalage par suite du problème de déperdition du à un mauvais système d'isolation.

\section{CONCLUSION}

Le distillateur solaire est un dispositif réalisable facilement avec des matériaux locaux, ce qui lui donne l'avantage d'être facilement exploitable pour des utilisateurs 
peu qualifiés on atteint des températures où la distillation et la condensation devient possible.

La conception du prototype de capteur photothermique avec l'absorbeur en film de polymère et les différents tests effectués ont montrés que les matériaux qui constituent le capteur ont une influence sur la distillation de l'eau saumâtre et par conséquent sur la quantité d'eau produite qui reste encore insignifiante pour ce modèle de prototype. Des efforts ont été envisagés pour la conception d'autres modèles de distillateurs en vue d'augmenter le rendement en améliorant les propriétés des matériaux.

L'énergie thermique disponible à partir d'un tel capteur est équivalente à l'énergie incidente diminuée des pertes dues à la réflexion, la réémission du rayonnement, la convection et la conduction.

La distillation dépend de plusieurs paramètres dont l'intensité de l'éclairement incident qui est variable en fonction du temps d'exposition, de la latitude du lieu et de l'état du ciel et la nébulosité (nuages). Elle dépend des propriétés absorbantes du film noir et des propriétés de transmission du verre et particulièrement du système d'isolation.

La température atteinte au niveau de l'absorbeur est de $66.6{ }^{\circ} \mathrm{C}$, que nous avons estimée suffisante pour le fonctionnement du système. En période nocturne, le stockage d'une quantité d'énergie absorbée au fil du soleil par la couche d'eau inférieure a permis au système de fonctionner pour distiller une quantité supplémentaire d'eau.

La différence de température entre l'absorbeur et le vitrage conduit au fonctionnement du capteur elle est d'autant plus grande que la quantité d'eau distillée produite est plus grande. Le débit de production d'eau ne dévient mesurable que lorsque le gradient de température entre l'absorbeur et le vitrage avoisine $10^{\circ} \mathrm{C}$. Nous avons constaté qu'a des périodes où le vent soufflant même à faible vitesse contribue au refroidissement de la vitre et par conséquent à une diminution de la température, ce qui accentue le processus de condensation de vapeur.

Les résultats de la distribution de la température ont montrés que la température n'est pas uniformément répartie selon les dimensions du capteur et dépend de l'isolation externe. La répartition axiale de la distribution de la température tout au long du distillateur montre une concentration de la température dans la zone tampon. Les résultats sont en accord avec ceux obtenus expérimentalement, malgré un décalage par suite du problème de déperdition du à un mauvais système d'isolation en absence de la réflexion par film d'aluminium. 
L'étude peut être étendue en améliorant les caractéristiques physiques des matériaux par le choix des surfaces sélectives pour la transmission du rayonnement et le choix du fluide caloporteur comme l'air pour le séchage par ventilation forcé ou le chauffage.

\section{REMERCIEMENTS}

Les auteurs remercient les contributions de la part des chercheurs de l'université de Tlemcen et des techniciens de laboratoire pour la réalisation du prototype de capteur thermique et remercient l'étudiante ayant choisi le sujet

\section{REFERENCES}

[1] Ghaffour N. (2008). Ressources en eau et dessalement en Algérie, Workshop on R.O desalination process, engineering practices, MEDREC Research Centre, Oman, Blida university of Algeria, Tipaza. 197 -209p.

[2] Bandelier et Deranzier J C, "Procédés thermiques de dessalement à haut rendement'' GRETH, Grenoble, groupe de recherche CLEFS CEA N 44 /2000 2001.

[3] Amara S. (2004). Optimisation d'un procédé économique de chauffage d'une habitation solaire: Application pour le site de Tlemcen, thèse de Magister, université de Tlemcen.

[4] Ainad T S. (2007). modélisation des transferts thermiques dans un distillateur solaire, PFE ingénieurs, Université Abou Bekr Belkaid - Tlemcen.

[5] Rauzier E. revue Phys. Appl. 1980, 15, 411- 415.

[6] Kahlen S and Gernot M. (2007). Wallner, Aging behavior of polymeric materials for solar thermal absorber applications, Proceedings of ISES Solar World Congress: Solar Energy and Human Settlement. 519 - 523p.

[7] Baba Ahmed N and Benmoussat A. (2007). Emissivity influence on the radiative thermal properties of opaque materials according to the temperature. ICRE '07, Conférence internationale sur les énergies renouvelables, Bejaia, 25-29p.

[8] Mokhtari F et Semmar D. Revue des Energies Renouvelables. 2001, 159-162. 


\section{DIAGNOSTIC SPECTROSCOPIQUE DE LAVTEMPÉRATURE ÉLECTRONIQUE DU PLASMAS PHOTOIONISE}

\section{RESUME}

Les énergies renouvelables telles que le solaire thermique sont des énergies accessibles pour le dessalement de l'eau, le recyclage et la production d'eau chaude. La chaleur est produite par effet de serre dans le capteur. C'est une forme d'énergie non polluante en accord avec le développement durable.

Dans cet article la conception du prototype de capteur photothermique avec l'absorbeur en film de polymère, les propriétés de rayonnement des matériaux, l'analyse de différentes déperditions d'énergie dans le système et le bilan énergétique seront présentés. L'évolution du rayonnement solaire incident sur le plan horizontal du capteur et la distribution de la température sont étudiées.

Les résultats ont montrés que la température obtenue par conversion thermique dépend des caractéristiques absorbantes du film polymère, de l'intensité de rayonnement et du temps d'exposition.

Mots clés : film, énergie solaire, effet de serre, conception, radiation, éclairement.

\section{How to cite this article}

Benmoussat A, Baba Ahmed N, Ainad Tabet S and Belahcene B. Study on the energetic parameters in a photothermic sensor with black polymeric film. J Fundam Appl Sci. 2010, 2(1), 1-11. 